\title{
Endometriose Cutânea Umbilical: Relato de Caso
}

\author{
Mário Aurélio Borges Fidelis', Chan I Thien', Bianca Gonçalves Varella Benjamim', Larissa Saboya Barbosa de Azevedo', \\ Mairan Santos Steffen', Maria de Fátima Guimarães Scotelaro', Arles Martins Brotas \\ 'Setor de Dermatologia, Hospital Universitário Pedro Ernesto, Rio de Janeiro, Brasil
}

RESUMO - PA endometriose caracteriza-se pela presença de implante ectópico de tecido endometrial, normalmente funcionante e hormono-sensível. O acometimento extrapélvico pode ocorrer em cerca de $12 \%$ das mulheres com a afecção. Endometriose umbilical foi relatada como representando cerca de $0,4 \%$ a $4,0 \%$ de todas as endometrioses e $30 \%$ a $40 \%$ dos casos de endometriose cutânea. Trata-se de um caso raro de uma doença que apresenta diagnósticos diferenciais importantes com outras afecções papulonodulares umbilicais, que podem ser primárias ou secundárias, neoplásicas ou inflamatórias. Relata-se o caso de uma paciente sem comorbidades, com diagnóstico de Endometriose cutânea umbilical, baseado na anamnese, exame clínico e histopatológico, que apresentou boa evolução após a exérese completa da lesão. O diagnóstico definitivo é histopatológico, mas pode ser complementado pela imunohistoquímica assim como a ultrassonografia, tomografia computorizada e ressonância magnética de abdómen. A excisão cirúrgica é a terapêutica de eleição.

PALAVRAS-CHAVE - Doenças da Pele; Endometriose; Umbigo.

\section{Umbilical Cutaneous Endometriosis: Case Report}

ABSTRACT - Endometriosis is characterized by the presence of ectopic implantation of endometrial tissue, normally functioning and hormone-sensitive. Extrapelvic involvement can occur in about $12 \%$ of women with the condition. Umbilical endometriosis has been reported to account for about $0.4 \%$ to $4.0 \%$ of all endometriosis and $30 \%$ to $40 \%$ of cases of cutaneous endometriosis. It is a rare case of a disease that presents important differential diagnosis with other umbilical papular disorders, which may be primary or secondary, neoplasic or inflammatory. This paper reports the case of a patient with no comorbidities, with a diagnosis of umbilical cutaneous endometriosis, based on anamnesis, clinical and histopathological examination, showing a good evolution after complete excision of the lesion. The definitive diagnosis is based on histopathology, but immunohistochemistry may be helpful as well as abdomen ultrasound, computed tomography and magnetic resonance imaging. Surgical management is the preferred treatment. KEYWORDS - Endometriosis; Skin Diseases; Umbilicus.

\section{INTRODUÇÃO}

A endometriose caracteriza-se pela presença de implante ectópico de glândulas e estroma endometrial, normalmente funcionante e hormono-sensível. ${ }^{1}$ É uma doença comum, geralmente crônica e benigna, que afeta frequentemente os órgãos pélvicos. ${ }^{2}$ Acomete em torno de $5 \%$ a $10 \%$ das mulheres em idade reprodutiva. ${ }^{3} \mathrm{O}$ envolvimento extrapélvico pode ocorrer em cerca de $12 \%$ das mulheres com a afecção. ${ }^{4}$

Endometriose umbilical foi relatada como representando cerca de $0,4 \%$ a $4,0 \%$ de todas as endometrioses e $30 \%$ a $40 \%$ dos casos de endometriose cutânea. ${ }^{5}$

Apesar da localização típica na região umbilical, pela raridade da manifestação, o diagnóstico muitas vezes é esquecido pelo dermatologista. A histopatologia é essencial e o método de escolha para o diagnóstico. A imunohistoquímica pode auxiliar no diagnóstico assim como ultrassonografia, ressonâcia magnética (RM) e tomografia computorizada (TC) do abdomen. A raridade dessa apresentação cutânea e a importância do diagnóstico diferencial nas afecções umbilicais foram os motivos desta comunicação.

\section{CASO CLÍNICO}

Paciente do sexo feminino, 42 anos, fototipo II, casada, moradora no Rio de Janeiro, sem comorbidades. Apresentava dor e eritema na região umbilical há dois anos, com agravamento durante o período menstrual. Há 8 meses havia notado presença de nódulo umbilical, que evolviu com crescimento lento e progressivo. Negava história de sangramento local.
Correspondência: Mário Aurélio Borges Fidelis

Hospital Universitário Pedro Ernesto - HUPE/UERJ - Setor de Dermatologia

Rua Boulevard 28 de Setembro, 77, Vila Isabel

Rio de Janeiro, 20551-030, Brasil

E-mail: marioaureliobfidelis@hotmail.com

DOI: https://dx.doi.org/10.29021/spdv.76.3.885
Recebido/Received

14 Janeiro/January 2018

Aceite/Accepted

18 Abril/April 2018 


\section{Caso Clínico}

Apresentava ciclos menstruais regulares associados à dismenorreia. Refere um parto por cesariana há 20 anos e um vaginal há 13 anos, sem abortamentos. Negava tabagismo e etilismo, ou outras intervenções cirúrgicas.

Ao exame físico, notava-se lesão nodular normocrómica, firme, aderente, medindo cerca de $2 \mathrm{~cm}$ de diâmetro, localizada na região umbilical, sem presença de sangramento ou sinais flogísticos. $\bigcirc$ exame histopatológico obtido por excisão cirúrgica completa do nódulo mostrou a derme com estroma mixóide fibrovascular típico com extravazamento de eritrócitos e hemossiderina, além de glândulas endometriais tortuosas revestidas por um epitélio colunar pseudoestratificado com secreção ativa. Não foram realizados outros exames prévios, como ultrassonografia e/ou ressonância magnética, pois optou-se pela imediata ressecção cirúrgica completa, posto que as dimensões da lesão a permitiram. Após exérese completa da lesão, a paciente foi encaminhada para acompanhamento na ginecologia para investigar a presença de outros implantes endometriais. A paciente evoluiu assintomática e sem recidiva após 1 ano de seguimento com a Dermatologia.

\section{DISCUSSÃO}

A endometriose é definida como a presença de tecido endometrial fora da cavidade uterina. Implantes extra-pélvicos podem ocorrer em $12 \%$ dos casos de endometriose. ${ }^{4} \mathrm{~A}$ incidência da endometriose cutânea umbilical é baixa, aproximadamente de $0,5 \%$ a $1 \%$ do total dos casos. ${ }^{6}$ Porém é o sítio mais comum da endometriose cutânea primária, pois representa, hipoteticamente como uma cicatriz fisiológica e tendo uma predilecção por implantes endometriais ectópicos. ${ }^{7}$

Existem dois tipos de endometriose, de acordo com a sua

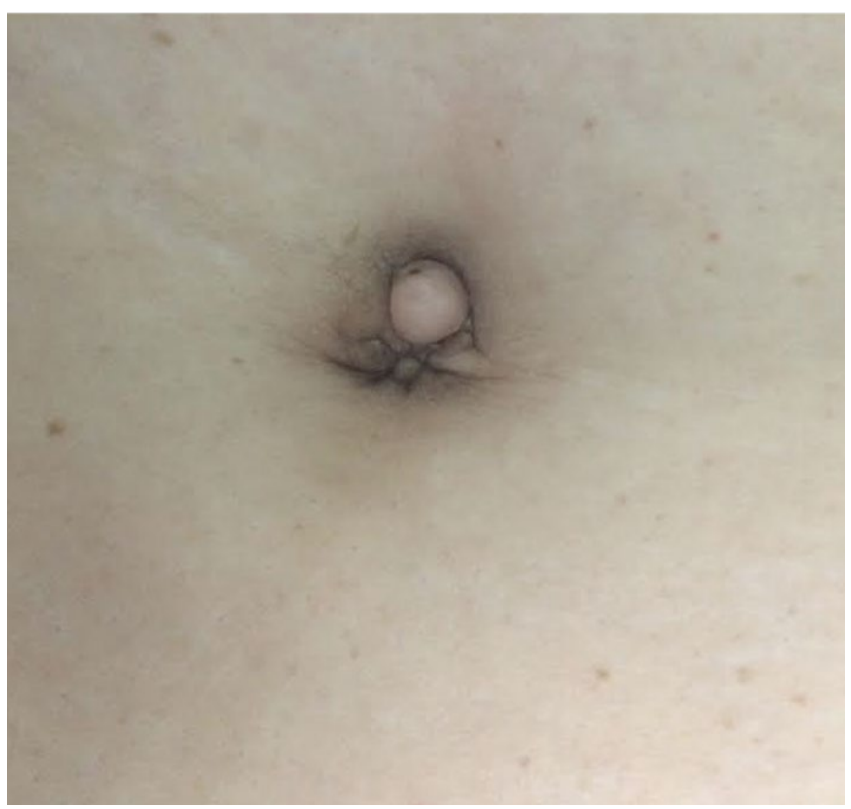

Figura 1 - Lesão nodular normocrómica com leve eritema, firme, aderente, medindo cerca de $2 \mathrm{~cm}$ de diâmetro, localizada na região umbilical.

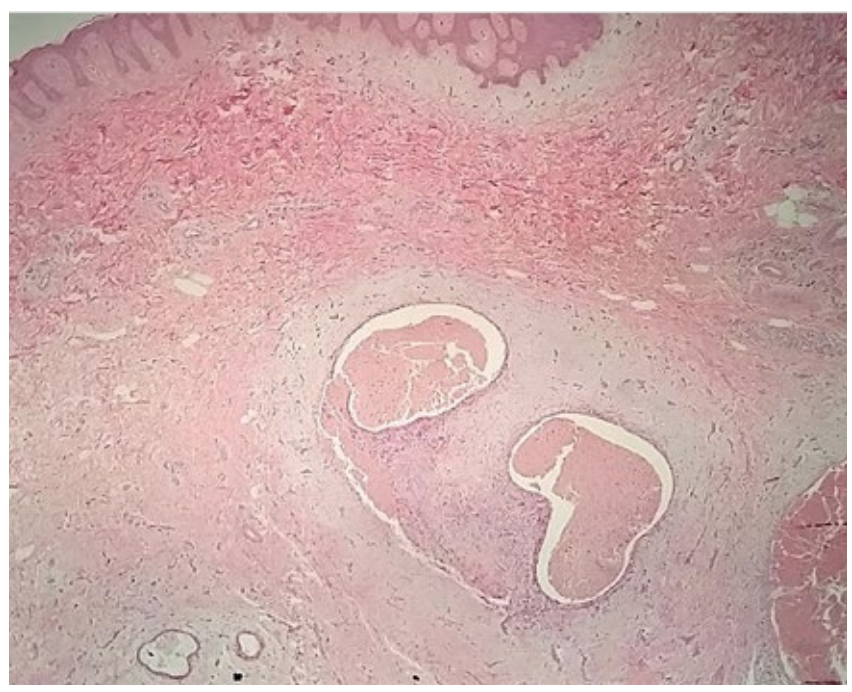

Figura 2 - Exame histopatológico $(H \& E ; \times 10)$ de biópsia do nódulo umbilical: derme com estroma mixóide fibrovascular típico associado a glândulas endometriais tortuosas.

etiologia: primária e secundária. A endometriose secundária é a mais comum e surge após procedimentos cirúrgicos no abdómen e pelve, como cesarianae laparotomia. ${ }^{8}$ A endometriose primária surge espontaneamente e não está relacionada a cirurgias prévias. ${ }^{8} \mathrm{O}$ caso relatado revelou endometriose cutânea umbilical em paciente com passado de cirurgia ginecológica.

A localização cutânea é rara, porém deve ser sempre pensada pelos dermatologistas principalmente quando estamos diante de lesões que se modificam com o período menstrual como na paciente em questão. ${ }^{9}$

Embora a etiologia não seja completamente entendida, existem várias teorias para explicar como o tecido endometrial migra do útero para a pele. Em 1920, Sampson formulou a hipótese de menstruação retrógrada. No entanto, existem várias outras teorias para o desenvolvimento de endometriose, incluindo metaplasia celómica, disseminação iatrogénica e linfática ou hematogénica. ${ }^{8}$ Como a paciente apresentava dismenorréia que poderia sugerir a presença de endometriose pélvica, além disso, tinha história de cesariana, não foi possível definir ao certo, qual mecanismo implicado na fisiopatogenia. Pode ter surgido após a translocação iatrogénica de massas endometriais para região umbilical, durante cirurgia cesariana ou pela teoria da menstruação retrógrada,onde o tecido endometrial ectópico é transmitido retrogradamente durante a menstruação pelas trompas para o local onde a endometriose está instalada.

O início tardio dos sintomas da endometriose cutânea após a cirurgia é uma das razões para o diagnóstico incorreto. $O$ período médio entre o procedimento e o início dos sintomas foi descrito na literatura como sendo de 4,5 anos a 5,72 anos. ${ }^{10}$ Entretanto, alguns autores descrevem o início precoce dos sintomas após 3 meses ou um início tardio após 10 anos. $^{10}$ No caso relatado, esse período foi de 20 anos. 


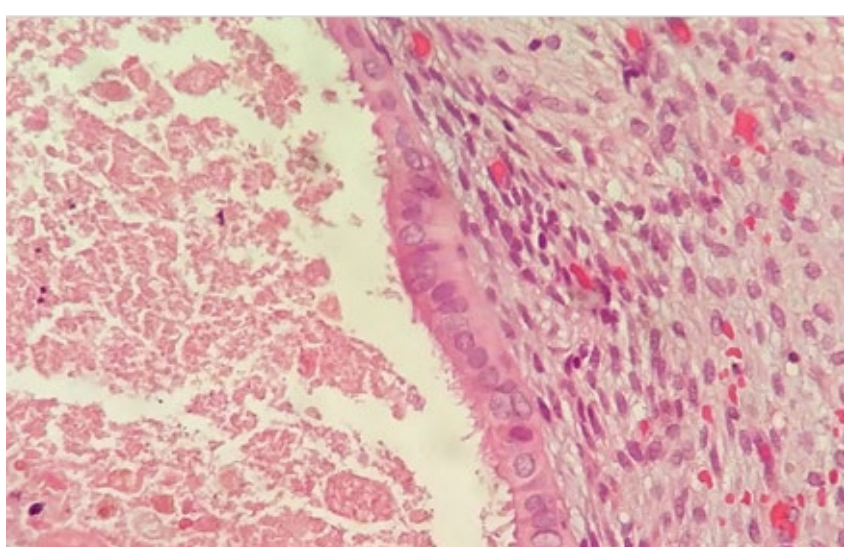

Figura 3 - Detalhe do exame histopatológico (H\&E; x40) onde se observa epitélio colunar pseudoestratificado com secreção ativa semeIhante à das glândulas apócrinas, à direita observa-se extravazamento de eritrócitos.

A endometriose umbilical faz diagnóstico diferencial com diversas afecções, incluindo doenças graves, como metástase umbilical de neoplasias do trato gastrointestinal (nódulo da irmã Maria José), e ainda, melanoma nodular, hérnia, pólipo fibroepitelial, granuloma piogénico, quisto e tumores anexiais. ${ }^{7}$ Em nosso caso, como a paciente apresentava lesão que se modificava de acordo com o ciclo menstrual e dismenorréia prévia, pensamos como primeira hipótese, endometriose cutânea.

Embora o diagnóstico pré-operatório seja amplamente dependente das características de imagem, o diagnóstico histopatológico isoladamente pode ser considerado como no caso em questão. " O gold standard para o diagnóstico de endometriose cutânea é o exame histopatológico. ${ }^{2,12}$ Nas lesões cutâneas podem ser observados espaços glandulares irregulares, circulares, alongados ou angulares, na derme reticular ou hipoderme, circundados por estroma altamente vascular e celular, semelhante ao do endométrio funcionante. ${ }^{12} \mathrm{O}$ aspecto histológico corresponde ao endométrio uterino nas fases proliferativa e secretória. ${ }^{5} \mathrm{~A}$ imunohistoquímica pode auxiliar no diagnóstico assim como ultrassonografia e RM/TC de abdómen. Relatórios recentes têm mostrado que a dermatoscopia também pode ser útil na investigação. ${ }^{8} \mathrm{Na}$ literatura, estão descritos achados como coloração vermelha homogénea, distribuída regularmente na lesão, clareando na periferia. Dentro dessa pigmentação usual algumas pequenas estruturas vermelhas globulares são descritas, as quais são referidas como "atóis vermelhos". ${ }^{13}$ Após uma correlação dermatoscópica-histopatológica foi visto que esses "atóis vermelhos" corresponderiam a glândulas irregulares contendo eritrócitos, encravados em estroma celular e o aspecto vermelho homogéneo corresponderia a um estroma vascular com aparência mixóide, contendo eritrócitos extravasados. ${ }^{13}$

Em relação ao tratamento, a excisão cirúrgica simples é a escolha, e deve ser ampla para garantir a cura completa da doença. ${ }^{3}$ A terapia hormonal com antagonistas da hormona libertadora de gonadotrofinas, anticoncepcional, gestrinona e danazol podem ser usados antes da excisão cirúrgica para diminuir o tamanho da lesão e aliviar sintomas caso estejam presentes. ${ }^{11} \mathrm{O}$ prognóstico da endometriose cutânea é bom. Recorrências são incomuns. No entanto, a transformação maligna tem sido relatada na literatura e deve ser suspeitada em lesões recorrentes ou de crescimento rápido. ${ }^{14} \mathrm{Em}$ até aproximadamente $1 \%$ dos endometriomas podem sofrer transformação maligna tipicamente para carcinoma endometrioide ou de células claras. ${ }^{11}$

Deve-se sempre investigar a possibilidade de coexistência de endometriose genital e/ou abdominal, o que piora o prognóstico, assim como modifica o tratamento, que, nesse caso, deve ser associado à terapia hormonal para alívio dos sintomas se presentes e evitar recorrência. ${ }^{5} \mathrm{~A}$ paciente em questão teve a confirmação diagnóstica antes da realização de exames de imagem por isso encaminhamos a mesma para acompanhamento no setor de Ginecologia para dar continuidade à pesquisa de outros implantes endometriais. Após o procedimento cirúrgico, não apresentou recidiva ou qualquer intercorrência em 1 ano de acompanhamento na Dermatologia.

\section{CONCLUSÃO}

A endometriose umbilical é, portanto, afecção rara, mas deve ser considerada no diagnóstico diferencial nos casos de mulheres em idade fértil que apresentem lesão na cicatriz umbilical, mesmo que assintomática. O diagnóstico definitivo é histopatológico, e a excisão cirúrgica é a terapêutica de eleição.

Conflitos de interesse: Os autores declaram não possuir conflitos de interesse.

Suporte financeiro: $O$ presente trabalho não foi suportado por nenhum subsídio ou bolsa.

Confidencialidade dos dados: Os autores declaram ter seguido os protocolos do seu centro de trabalho acerca da publicação dos dados de doentes.

Protecção de pessoas e animais: Os autores declaram que os procedimentos seguidos estavam de acordo com os regulamentos estabelecidos pelos responsáveis da Comissão de lnvestigação Clínica e Ética e de acordo com a Declaração de Helsínquia da Associação Médica Mundial.

Consentimento dos doentes: Obtido.

Conflicts of interest: The authors have no conflicts of interest to declare.

Financing Support: This work has not received any contribution, grant or scholarship.

Confidentiality of data: The authors declare that they have followed the protocols of their work center on the publication of data from patients.

Protection of human and animal subjects: The authors declare that the procedures followed were in accordance with the regulations of the relevant clinical research ethics committee and with those of the Code of Ethics of the World Medical Association (Declaration of Helsinki).

Patients consent: Obtained. 


\section{Caso Clínico}

\section{REFERÊNCIAS}

1. Steck WD, Helwig EB. Cutaneous endometriosis. JAMA. 1956; 191: 167-70.

2. Fernández-Aceñero MJ, Córdova S. Cutaneous endometriosis: review of 15 cases diagnosed at a single institution. Arch Gynecol Obstet. 2011; 283:1041-4.

3. Agarwal A, Fong YF. Cutaneous endometriosis. Singapore Med J. 2008; 49:704-9.

4. Choi SW, Lee HN, Kang SJ, Kim HO. A case of cutaneous endometriosis in postmenopausal woman receiving hormonal replacement. J Am Acad Dermatol. 1999; $41: 327-9$.

5. Loh S-H, Lew B-L, Sim W-Y. Primary cutaneous endometriosis of umbilicus. Ann Dermatol. 2017; 29:621 5.

6. Pramanik SR, Mondal S, Paul S, Joycerani D. Primary umbilical endometriosis: A rarity. J Hum Reprod Sci. 2014; 7: 269-71.

7. Van den Nouland D, Kaur M. Primary umbilical endometriosis: a case report. Facts Views Vision Obgyn. 2017; 9:115-9.

8. Lopes Mattos S, Pereira Christo AG. Primary umbilical endometriosis. Surg Cosmet Dermatol. 2015; 7/3 Suppl 1):S43-6.

9. Fernández Vozmediano JM, Armario Hita JC, Cuevas Santos J. Cutaneous endometriosis. Int J Dermatol. 2010; 49: 1410-2.

10. Kaya BA, Emrah C, Cem K, Serel S. Cutaneous endometriosis. Rev Bras Cir Plást. 2012; 27: 493-5.

11. Kocher M, Hardie A, Schaefer A, McLaren T, Kovacs $M$. Cesarean-section scar endometrioma: a case report and review of the literature. J Radiol Case Rep. 2017; 11:16-26.

12. Tidman MJ, MacDonald DM. Cutaneous endometriosis: a histopathologic study. J Am Acad Dermatol. 1988; 18:373-7.

13. Jaime TJ, Jaime TJ, Ormiga $P$, Leal F, Nogueira OM, Rodrigues N. Endometriose umbilical: relato de um caso e seus achados dermatoscópicos. An Bras Dermatol. 2013;88:101-4.

14. Terada S, Miyata Y, Nakazawa H, Higashimori T, Arai T, Kikuchi $Y$, et al. Immunohistochemical analysis of an ectopic endometriosis in the uterine round ligament. Diagn Pathol. 2006;1:27. 